\title{
Vieillissement et intégrité de la peau
}

> La peau est un organe sentinelle, soumis au vieillissement chronologique et environnemental qui fragilise sa structure et ses fonctions. La fonction barrière de la peau, ses propriétés élastiques et de résistance, ainsi que sa réactivité vasculaire sont atteintes par le vieillissement dans les compartiments épidermiques, dermiques et vasculaires. Les progrès de la recherche ont permis de révéler des processus biologiques sous-jacents, qui peuvent être ciblés par des approches médicamenteuses topiques ou globales à base notamment d'anti-oxydants ou de sénolytiques. Ces stratégies anti-âge pourront contribuer à restaurer, au moins en partie, l'intégrité fonctionnelle de la peau âgée. <

Bien que dans certaines cultures l'âge soit associé à une forme de sagesse et donc impose le respect, le vieillissement est le plus souvent une source d'inquiétudes dans nos sociétés occidentales en quête permanente de jeunesse. La peau étant en contact direct avec l'environnement, elle est directement exposée aux agressions du milieu extérieur et, notamment, aux rayons solaires ultraviolets (UV) responsables du photo-vieillissement qui accélère les effets de l'âge. De fait, la peau reflète, tel un miroir, l'intégrité physiologique de l'ensemble de nos fonctions vitales. Cet organe «sentinelle», parcouru par un important réseau de vaisseaux sanguins et de fibres nerveuses, est constitué de trois couches distinctes et superposées: l'épiderme, le derme et l'hypoderme. Des manifestations visibles du vieillissement, telles que des rides, des taches, une sécheresse cutanée, une perte d'élasticité ou encore une dilatation des petits vaisseaux sanguins apparaissent à la surface de notre peau avec le temps. Avec l'allongement de la durée de vie, la lutte contre le vieillissement et ses conséquences est devenue un enjeu sociétal majeur. Le contrôle du vieillissement cutané est clairement un défi scientifique et économique de premier plan.

Même si des soins à base de produits naturels ont été utilisés de tout temps pour entretenir la jeunesse de

\section{De la biologie cutanée aux stratégies anti-âge}

Julie Rorteau, Fabien P. Chevalier, Bérengère Fromy, Jérôme Lamartine

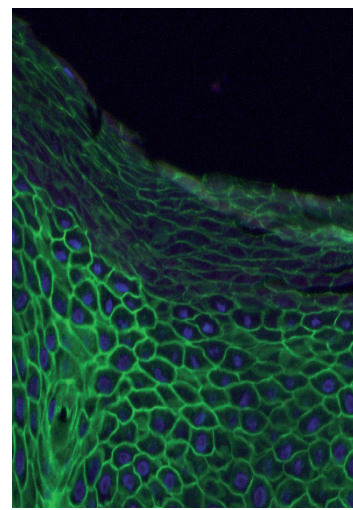

Équipe Intégrité fonctionnelle du tissu cutané (SKIN). Laboratoire de biologie tissulaire et d'ingénierie thérapeutique (LBTI), CNRS UMR5305, Université Lyon I,

7 passage du Vercors, 69367 Lyon Cedex 07, France. Jerome.lamartine@univ-lyonl.fr

la peau, on peut envisager aujourd'hui de lutter plus efficacement contre le vieillissement de la peau grâce à une meilleure compréhension des mécanismes biologiques de ce dernier et de la physiologie du tissu cutané. À l'échelle cellulaire, de nombreux mécanismes et marques du vieillissement chronologique ou intrinsèque ont été décrits [1], ce qui ouvre la voie à des stratégies anti-âge ciblées reposant sur des connaissances scientifiques établies. Au-delà des désagréments physiques, c'est la fonctionnalité même du tissu cutané, première barrière naturelle entre le corps et l'environnement, qui est altérée par le vieillissement, pouvant aller jusqu'à des complications médicales. Restaurer la fonctionnalité de la peau âgée est un objectif majeur qui suscite de nombreux travaux de recherche, notamment dans notre équipe.

\section{Altérations de la fonction barrière de l'épiderme}

L'épiderme, couche la plus superficielle de la peau, a évolué, afin de constituer une barrière face à l'environnement. II assure en outre le maintien de l'hydratation, une capacité essentielle pour la survie des organismes terrestres. Cet épithélium pluristratifié comprend plusieurs types cellulaires : les cellules de Langerhans, qui garantissent la veille immunitaire, les mélanocytes, qui permettent la pigmentation de la peau, les cellules de Merkel, qui sont impliquées dans la sensation mécanique et, enfin, les kératinocytes, qui représentent à eux seuls environ $90 \%$ de la population épidermique, et qui vont permettre d'assurer la fonction barrière. Pour cela, les kératinocytes vont subir une différenciation progressive, par étapes, selon un axe vertical orienté de 


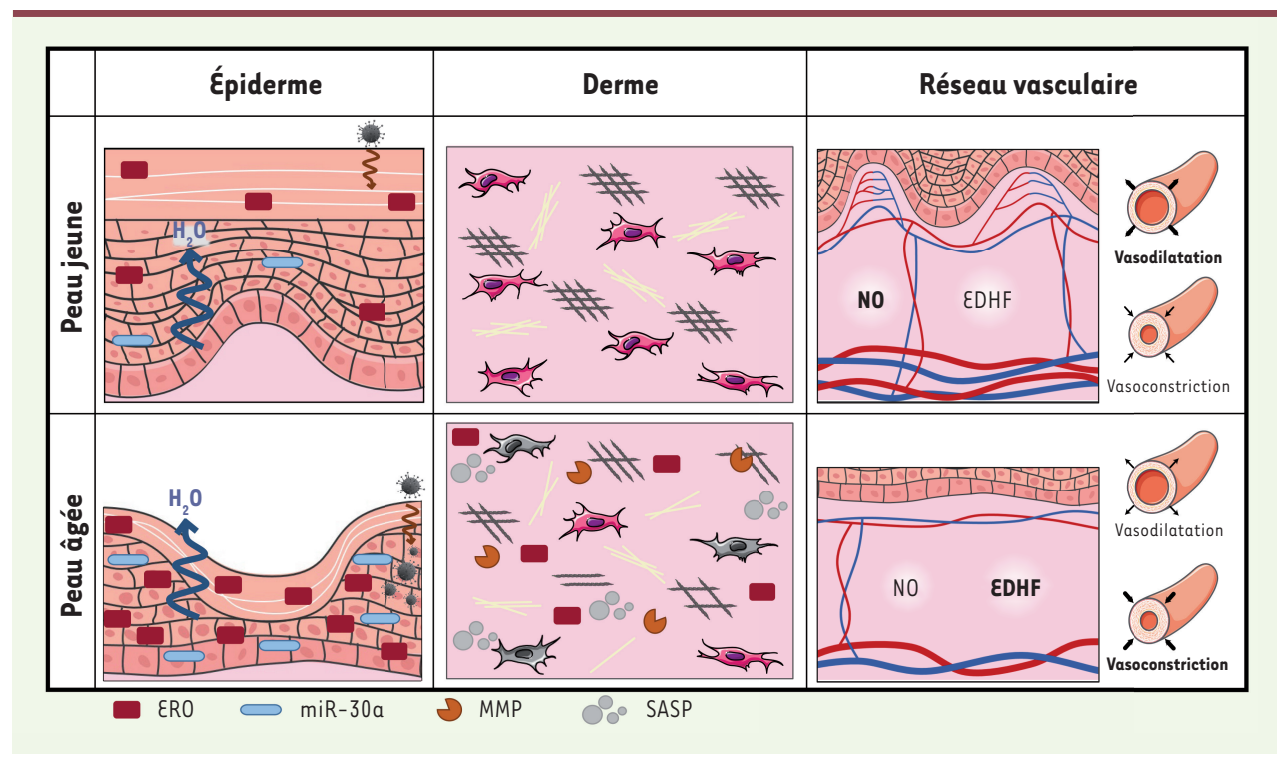

Figure 1. Représentations graphiques des principales modifications de l'épiderme, du derme et du réseau vasculaire liées au vieillissement chronologique. L'épiderme, le derme et de réseau vasculaire sont impactés différemment par le vieillissement cutané. 1) L'épaisseur de l'épiderme est réduite et les papilles dermiques s'aplatissent avec l'âge. La surexpression du miR-30a et l'accumulation d'espèces réactives de l'oxygène (ERO) dans le tissu âgé entraînent une perturbation de la différenciation kératinocytaire et de la barrière épidermique qui devient plus perméable à l'eau et aux infections microbiennes ; 2) Au sein du derme, l'accumulation d'ER0 et de métalloprotéases matricielles (MMP) entraîne la désorganisation des réseaux de collagène et de fibres élastiques. De plus, de nombreux fibroblastes entrent en sénescence et sécrètent des SASP (senescence-associated secretory phenotype) qui participent à la propagation du vieillissement dans le tissu ; 3) La taille des vaisseaux sanguins, ainsi que leur nombre sont réduits dans la peau âgée par rapport à la peau jeune. De plus, il y a un défaut de vasodilatation des vaisseaux directement lié à une perturbation de la voie du monoxyde d'azote (NO) ainsi qu'une surexpression d'un facteur hyperpolarisant dérivé de l'endothélium (EDHF). Adaptés de la banque d'image libre de droit de Servier Medical Art (https://smart.servier.com) sous licence creative commons.

l'intérieur vers l'extérieur. Au stade terminal de leur différenciation, les kératinocytes peuvent être assimilés à des cellules momifiées, appelées cornéocytes, et dépourvues de noyau et d'organites cellulaires. Au cours du vieillissement physiologique, l'épaisseur de l'épiderme diminue significativement, la jonction dermo-épidermique s'aplatit et la fonction barrière s'altère graduellement (Figure I) avec, pour conséquences immédiates, une susceptibilité accrue aux infections et une déshydratation exacerbée, due à une perte d'eau par voie transépidermique. L'amincissement de l'épiderme, associé à la dégénérescence de la fonction barrière, suppose une altération intrinsèque du processus de différenciation des kératinocytes avec l'âge. Contrairement à d'autres tissus, ni le nombre ni la fonctionnalité des cellules souches épidermiques ne semblent affectés par le vieillissement chronologique. En effet, dans une étude réalisée chez la souris, on observe que le nombre de cellules souches localisées dans les follicules pileux reste stable à 6 , 12, 18 ou 22 mois d'âge [2]. Toutefois, dans l'espace interfolliculaire de la couche basale de l'épiderme, les capacités prolifératives des progéniteurs kératinocytaires diminuent légèrement avec le temps [2]. Très récemment, une étude complémentaire, par analyse du transcriptome en cellule unique, a confirmé que les cellules souches épidermiques, ainsi que les progéniteurs, maintenaient leur identité cellulaire entre 2 mois et 24 mois chez la souris [3]. Il apparaît donc que le vieillissement chronologique perturbe l'engagement des cellules souches vers la différenciation épidermique. Depuis quelques années, l'hypothèse d'une érosion épigénétique avec l'âge suscite un intérêt croissant. De nombreuses études ont d'ores et déjà validé la corrélation entre le profil de méthylation de l'ADN et l'âge de l'individu, en particulier dans la peau, que ce soit chez l'homme ou chez d'autres mammifères [4]. Notre équipe a exploré un autre aspect épigénétique du vieillissement épidermique, en réalisant une étude exhaustive de l'expression des micro-ARN (miARN) dans les kératinocytes humains jeunes ou âgés [5]. Les miARN régulent simultanément l'expression de multiples gènes au niveau post-transcriptionnel. Ils influencent ainsi les destins cellulaires en affectant, entre autres, les balances prolifération/différenciation ou encore survie/apoptose [6]. Le miR-30a est le miARN dont l'expression dans les kératinocytes humains est la plus amplifiée avec l'âge. II est connu pour cibler des acteurs de l'autophagie, et notamment la bécline-1, dans les processus de tumorigenèse. Nous avons montré que la simple surexpression de ce miARN dans des kératinocytes jeunes, isolés de peau humaine, suffit à reproduire, dans un modèle organotypique d'épiderme reconstruit, les caractéristiques majeures du vieillissement, telles que l'augmentation de l'apoptose et un défaut de différenciation des kératinocytes, conduisant ainsi à une altération de la fonction barrière de ces cellules [5]. Dans l'épiderme, une dérégulation $\mathrm{du}$ processus autophagique des organites par miR-30a pourrait expliquer en partie le défaut de différenciation des kératinocytes observé avec l'âge (Figure 2). Nous 


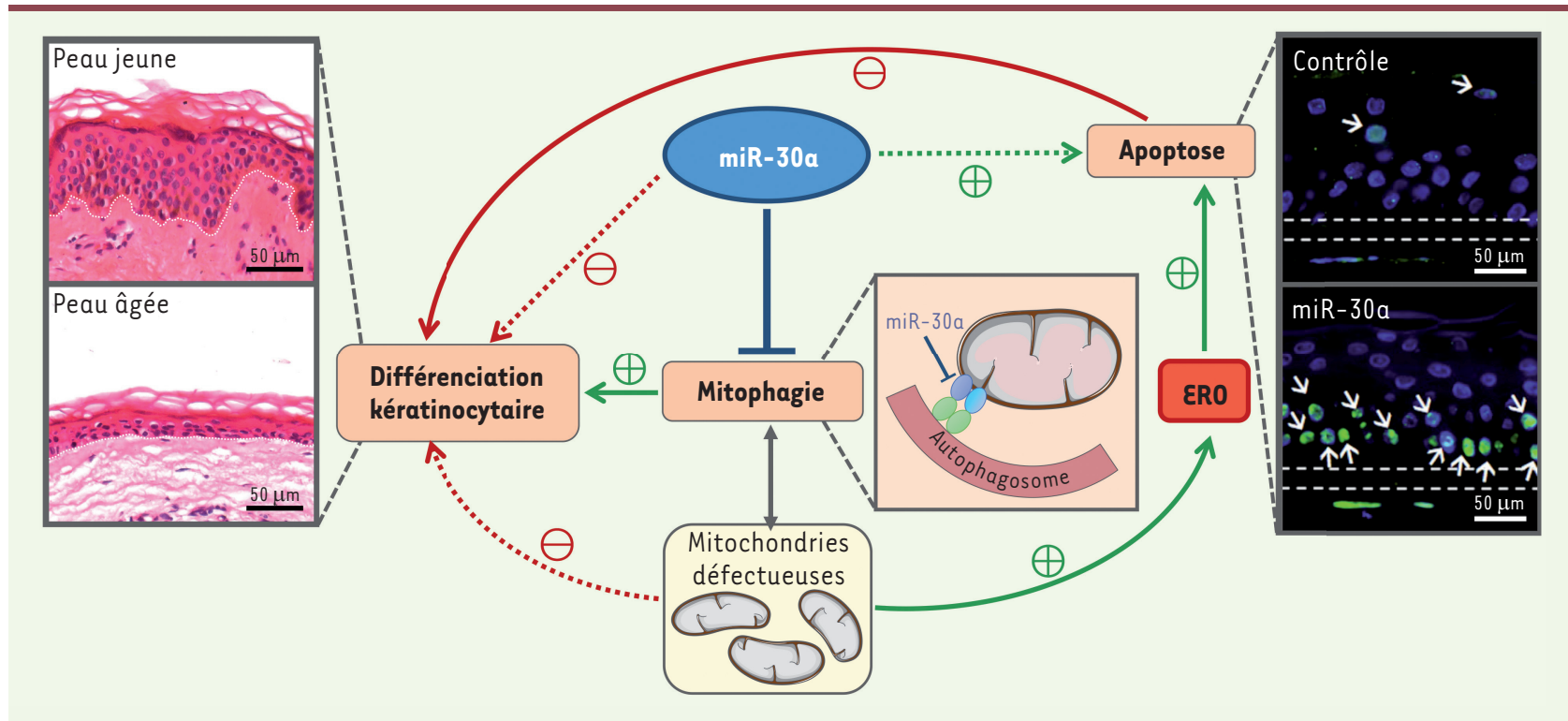

Figure 2. Implication du miR-30a dans le vieillissement épidermique. La surexpression du miR-30a dans un modèle organotypique d'épiderme reconstruit augmente considérablement le taux de kératinocytes en apoptose (marquage TUNEL vert, encadré de droite) et perturbe la différenciation normale des kératinocytes, reproduisant ainsi l'altération de la structure de l'épiderme observée avec l'âge (coloration hématoxyline/ éosine, encadré de gauche). Nos travaux actuels montrent que miR-30a cible des acteurs de la mitophagie. L'inhibition de la mitophagie entraîne une accumulation des mitochondries défectueuses et donc une augmentation de la concentration intracellulaire d'espèces réactives de l'oxygène (ERO). Parallèlement, la rétention des mitochondries perturbe le processus normal de différenciation kératinocytaire, conduisant ainsi à une

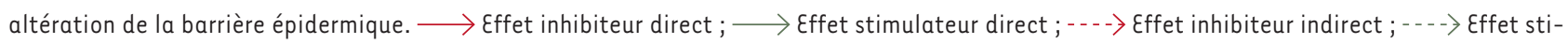
mulateur indirect. Adaptés de la banque d'image libre de droit de Servier Medical Art (https://smart.servier.com) sous licence creative commons.

avons montré que miR-30a cible un acteur central de la mitophagie. Cette hypothèse d'un défaut de mitophagie induite par ce miARN est par ailleurs renforcée par l'augmentation effective des espèces réactives de l'oxygène ( $(R 0)$ que l'on observe avec le vieillissement épidermique [7]. Or, il existe dans l'épiderme un gradient de facteurs antioxydants, avec une concentration maximale au niveau de l'enveloppe cornée [8], qui correspond au stade terminal de la différenciation des kératinocytes. La perturbation de la mitophagie associée au vieillissement engendrera ainsi simultanément une altération du métabolisme mitochondrial, avec une augmentation de la concentration locale des $E R O$ et une altération du processus de différenciation des kératinocytes, accompagné d'une diminution de la production des systèmes antioxydants, accélérant ainsi le vieillissement de l'épiderme et des autres compartiments de la peau.

\section{Altérations de la fonction dermique}

Au contraire de l'épiderme qui est composé de cellules jointives et serrées, le derme est majoritairement constitué d'une matrice extracellulaire (MEC). Les fibres de collagène sont le composant principal de cette matrice. Elles lui confèrent sa résistance aux chocs mécaniques. Les fibres élastiques, le deuxième élément fibreux de la $M E C$, permettent à la peau de reprendre sa forme initiale après une déformation. Enfin, les protéoglycanes et les glycosaminoglycanes
(GAG) constituent le gel dans lequel sont ancrés ces composants fibreux et offrent une résistance aux forces de compression. Ce gel représente également la plus grande réserve hydrique de la peau, notamment grâce à la très grande capacité de rétention d'eau de l'un des GAG, l'acide hyaluronique. Tous ces composants matriciels sont synthétisés et dégradés par les cellules résidentes du derme, les fibroblastes. Ces cellules sécrètent des collagénases et des protéases, ainsi que leurs inhibiteurs, afin de remodeler la MEC qui les entoure [9]. Chacun des composants du derme est affecté par le processus de vieillissement (Figure 1) [10]. En effet, le réseau dense et ferme des fibres de collagène est désorganisé dans la peau des personnes âgées [9], essentiellement en raison d'un déséquilibre de la balance entre synthèse et dégradation, résultant de l'augmentation de la sécrétion des collagénases de type MMP (matrix metalloproteinase) [11]. La cause principale de cette perturbation est l'augmentation des ERO dans la peau âgée, qui pourrait être associée à la dysfonction mitochondriale de l'épiderme. Le nombre de fibres élastiques diminue également avec le vieillissement physiologique, ce qui amplifie d'autant plus la lâcheté du réseau matriciel dermique, provoquant l'apparition des rides. 
L'altération de ce réseau fibreux entraîne en parallèle, une diminution de l'interaction des fibroblastes avec la MEC [10]. Cette perte de contact physique des cellules, associée aux nombreux stress qu'elles accumulent au cours du vieillissement physiologique, tels que des dommages à l'ADN, le stress oxydant ou encore l'exposition aux ultra-violets (UV), accroît le nombre de cellules qui entrent en sénescence [12]. Cet état particulier permet aux cellules de sortir du cycle cellulaire afin de ne pas transmettre de mutations à la génération suivante. Mais les cellules sénescentes résistent à l'apoptose, restent métaboliquement actives, et possèdent un sécrétome qui leur est propre, appelé SASP (senescence-associated secretory phenotype), qui va impacter leur environnement [13]. II a notamment été montré que des vésicules extracellulaires $(V \varepsilon)$, fortement impliquées dans les communications intercellulaires, participent à la propagation de ce SASP [14]. Le contenu de ces vésicules dont, en particulier, des miARN [6], est fortement modifié entre les cellules normales et les cellules sénescentes. Les signaux transmis aux cellules environnantes sont donc différents, conduisant petit à petit à l'accumulation de cellules sénescentes et au vieillissement généralisé du tissu.

Dans la peau, les fibroblastes du derme sont fortement touchés par ce phénomène. Plusieurs études ont déjà démontré que les VE sécrétées par les fibroblastes ont un effet direct sur les kératinocytes de l'épiderme via les miARN qu'elles contiennent. In vitro, les VE produites par les fibroblastes sénescents ralentissent la fermeture des blessures [15]. Il semblerait également que ces vésicules « sénescentes » soient moins efficaces pour soutenir la différenciation des kératinocytes que les $V \varepsilon$ de fibroblastes jeunes [16]. Ainsi, la multiplication des fibroblastes sénescents au cours du vieillissement concourt à l'altération de l'état fonctionnel des fibroblastes environnants: ils perturbent leurs communications, majoritairement au travers de la sécrétion de SASP et notamment de VE porteuses du message de vieillissement, avec les compartiments environnants, tels que l'épiderme ou le réseau vasculaire cutané. Le derme est en effet la structure nourricière essentielle de la peau, grâce aux vaisseaux sanguins qu'il contient. On comprend alors aisément que toute altération de la fonction dermique va également durablement affecter la fonction vasculaire.

\section{Altérations de la fonction vasculaire cutanée}

Dans la peau, le réseau vasculaire est disposé en deux plexus sousépithéliaux [17]. Outre I'homéostasie tissulaire locale, la modulation du flux sanguin cutané (vasodilatation/vasoconstriction) est fortement impliquée dans le contrôle de la thermorégulation, de la pression artérielle et de l'homéostasie circulatoire générale. Les cellules endothéliales des vaisseaux qui y sont présents, sécrètent des substances vasorelaxantes (monoxyde d'azote [NO], prostacycline, et un facteur hyperpolarisant dérivé de l'endothélium [EDHF]) ainsi que d'autres molécules vasoconstrictrices (endothélines-1 [ET-1], thromboxane $A 2$, prostaglandine $H 2$, anions superoxydes, et les composants du système rénine-angiotensine), donnant à l'endothélium un statut de tissu fonctionnel.
Le vieillissement est à l'origine de modifications anatomiques affectant la microcirculation cutanée, qui sont marquées par une diminution de la taille et du nombre de vaisseaux, en relation avec un défaut d'angiogenèse (Figure 1) [18]. Cette perte de la densité des vaisseaux nutritionnels cutanés et de la surface d'échange s'accompagne d'une désorganisation du réseau vasculaire, pouvant expliquer certaines altérations physiologiques caractéristiques de la peau âgée, notamment la pâleur, la diminution de la température cutanée, la réduction de l'érythème induit par les UV, la diminution de l'apport nutritionnel et la réduction de la réactivité vasculaire cutanée [19].

Le vieillissement diminue également les capacités fonctionnelles vasculaires de la peau en réponse à différents stimulus (thermiques, mécaniques, pharmacologiques). En revanche, la relaxation du muscle lisse vasculaire (vasodilatation indépendante de l'endothélium) semble préservée avec l'avancée en âge, même si une altération de ce muscle peut apparaître chez des sujets plus âgés. Il est ainsi admis que l'altération de la réactivité vasculaire cutanée qui accompagne le vieillissement est liée à une dysfonction endothéliale [20] impliquant plusieurs mécanismes (Figure 3). Avec l'âge, il existe en effet un déséquilibre entre les différents facteurs endothéliaux de relaxation et de contraction, sans que leurs rôles respectifs ne soient clairement établis [20]. Mais la majorité des données obtenues indique que cette dysfonction endothéliale liée à l'âge se produit en raison d'une altération de la voie responsable de la production du NO [21]. La réduction de la production du NO avec l'âge est liée à la diminution de l'expression et/ou de l'activité de la NOS (NO synthase) endothéliale, à l'origine de la baisse de la biodisponibilité du précurseur du NO (la L-arginine), de la carence en tétrahydrobioptérine, un cofacteur intervenant dans la synthèse du NO, et de l'augmentation de la production d'un inhibiteur endogène de la NOS (diméthylarginine asymétrique). Le NO résiduel produit peut néanmoins réagir avec un $\varepsilon R 0$, l'anion superoxyde $\left(0_{\dot{2}}^{-}\right)$, pour former du peroxynitrite $\left(\mathrm{ONOO}^{-}\right)$, ce qui entraîne des dommages oxydants dans les cellules endothéliales qui sont à l'origine de la diminution de la relaxation vasculaire [22]. L'état inflammatoire chronique de bas grade (ou inflammaging) va également compromettre la biodisponibilité en NO [23]. En parallèle, l'activité des cyclo-oxygénases (COX) ${ }^{1}$ diminue avec l'âge, avec pour conséquence, un déséquilibre en faveur des

\footnotetext{
${ }^{1}$ Le métabolisme de l'acide archidonique (produit par la phospholipase A2 à partir des phospholipides membranaires) par la voie de la cyclooxygénase (COX) donne naissance aux prostaglandines (PGE2, PGD2, PGH2, PGF2A), à la prostacycline (PGI2) et au thromboxane, à partir d'un même précurseur, la prostaglandine H2.
} 


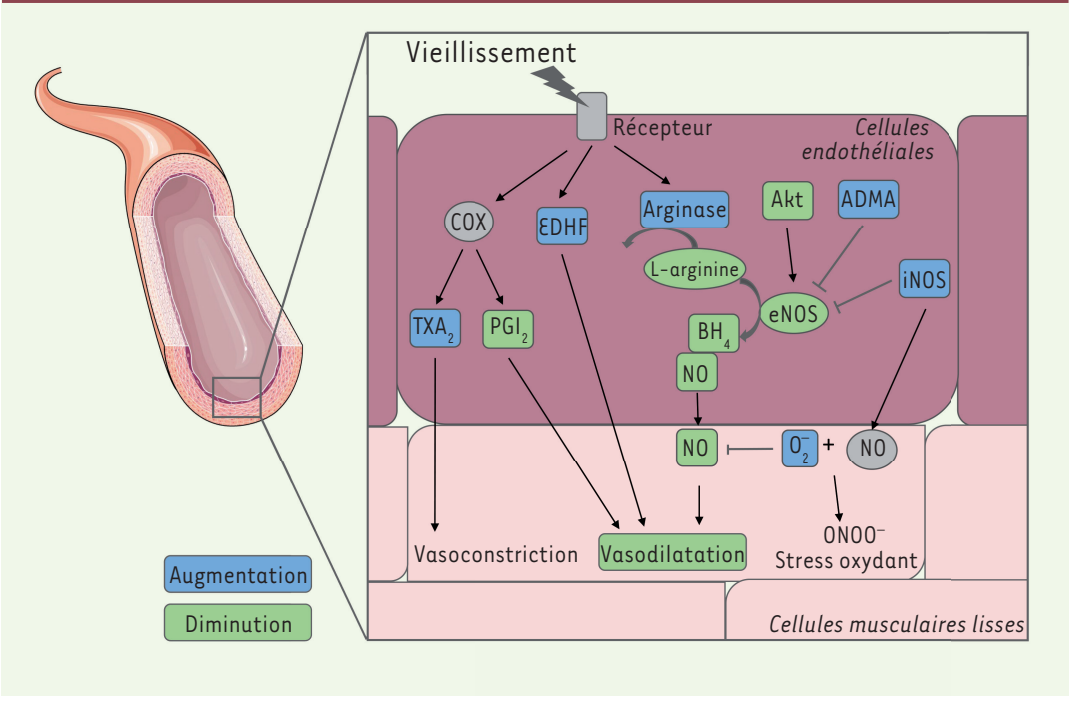

Figure 3. Principaux mécanismes impliqués dans l'altération de la vasodilatation endothéliumdépendante liée au vieillissement chronologique. Au cours du vieillissement, un déséquilibre s'opère entre les différents facteurs endothéliaux de relaxation et de contraction. Globalement, la production du NO diminue via la baisse de biodisponibilité de la NO synthase endothéliale (eNOS), de son précurseur (L-arginine) et de co-facteurs, tels que la tétrahydrobioptérine (BH4). De plus, le NO formé peut réagir rapidement avec le superoxyde pour former du peroxynitrite (ONOO-) et entraîner des dommages oxydants. Concernant les produits dérivés des cyclo-oxygénases (COX), l'âge va conduire à une augmentation des vasoconstricteurs (thromboxane A2) et à une diminution des vasodilatateurs (prostacycline PGI2). Malgré une augmentation de l'activité du facteur hyperpolarisant dérivé de l'endothélium ( $E D H F$ ), les capacités de vasodilatation diminuent. Adaptés de la banque d'image libre de droit de Servier Medical Art (https://smart.servier.com) sous licence creative commons.

vasoconstricteurs (thromboxane A2) par rapport aux vasodilatateurs (prostacycline [PGI2]) [24]. Les voies des endothélines-1 et le système rénine-angiotensine jouent ainsi un rôle dans la dysfonction endothéliale liée à l’âge.

La voie du NO et celle des prostaglandines (PGI2) interagissent entre elles: lorsqu'une de ces voies est inhibée ou altérée, la contribution de l'autre voie augmente. Au cours du vieillissement, l'EDHF (endothelium-derived hyperpolarizing factor) semble intervenir lorsque les 2 voies (NO et PGI2) sont déficientes [25]. Chez les souris âgées, en compensant partiellement l'altération des voies du NO et des prostaglandines, l'EDHF permet en effet de préserver une vasodilatation cutanée à une pression suffisante, bien que celle-ci soit réduite par rapport à celle observée chez des souris jeunes. À noter que l'instabilité génomique des cellules endothéliales et la baisse de production des œstrogènes peuvent également contribuer à la dysfonction endothéliale au cours du vieillissement.

\section{Conséquences des défauts vasculaires cutanés chez la personne âgée}

Les défauts vasculaires cutanés associés au vieillissement diminuent la capacité à dissiper la chaleur [19] : la personne âgée est ainsi en danger lors des épisodes de canicule, avec un impact sociétal considérable.

Terrien, nous sommes en permanence en appui sur une surface cutanée. Cette situation, à laquelle nous sommes adaptés, est à l'origine de lésions ischémiques induites par la pression vis-à-vis desquelles la peau est protégée [26]. Cette capacité de défense de la peau repose sur des mécanismes neuro-vasculaires dépendant de l'endothélium [27]. Au cours du vieillissement, la peau devient fragile et la dys- fonction endothéliale liée à l'âge s'accompagne d'une diminution de ses capacités de défense, qui s'aggrave lorsque la personne âgée présente une neuropathie sensorielle périphérique, ce qui la prédispose aux escarres [28, 29]. Lésion ischémique, l'escarre détériore la qualité de vie des sujets et entraîne des complications socio-économiques très importantes, induisant une surcharge en soins infirmiers, des traitements onéreux, et une prolongation de séjour en structure hospitalière.

\section{Stratégies anti-âge : vers une restauration de la fonctionnalité du tissu cutané ?}

Les stratégies anti-âge consistent à limiter les effets du vieillissement, notamment aux niveaux dermique, vasculaire et épidermique (Tableau I). Ces approches sont multiples et impliquent des stratégies variées, aussi bien pharmacologiques, topiques ou générales, qu'invasives. Nous nous limiterons dans cette revue, à quelques stratégies pharmacologiques ciblant des mécanismes moléculaires ou cellulaires connus pour être impactés par le vieillissement dans la peau. Les approches plus invasives sont présentées dans la seconde Synthèse sur le vieillissement de la peau proposée dans ce numéro [41] $(\rightarrow)$.

$(\rightarrow)$ Voir la Synthèse de A. Bensussan et L. Michel, page 1163 de ce numéro

L'application topique de molécules anti-oxydantes, comme les vitamines $C, B 3$ et $\varepsilon$, les polyphénols ou les flavonoïdes, est une stratégie de choix, notamment pour lutter contre le photo-vieillissement, générateur 


\begin{tabular}{|c|c|c|c|c|c|}
\hline Compartiment & $\begin{array}{l}\text { Altération } \\
\text { fonctionnelle }\end{array}$ & $\begin{array}{l}\text { Conséquences } \\
\text { cliniques sur } \\
\text { l'intégrité de la peau }\end{array}$ & $\begin{array}{l}\text { Processus } \\
\text { biologiques } \\
\text { sous-jacents }\end{array}$ & $\begin{array}{l}\text { Mécanismes } \\
\text { moléculaires } \\
\text { impliqués }\end{array}$ & $\begin{array}{l}\text { Stratégies } \\
\text { anti-âge }\end{array}$ \\
\hline Épiderme & $\begin{array}{l}\text { Perte } \\
\text { de la fonction } \\
\text { barrière }\end{array}$ & $\begin{array}{l}\text { Déshydratation } \\
\text { Sensibilité accrue } \\
\text { aux infections }\end{array}$ & $\begin{array}{l}\text { Défaut } \\
\text { de différenciation } \\
\text { des kératinocytes }\end{array}$ & $\begin{array}{l}\text { Inhibition } \\
\text { de la mitophagie } \\
(m i R-30 a ?)\end{array}$ & $\begin{array}{l}\text { Molécules anti- } \\
\text { oxydantes } \\
\text { (vit. C-B3-E, } \\
\text { polyphénols, } \\
\text { flavonoïdes) }\end{array}$ \\
\hline Derme & $\begin{array}{l}\text { Perte d'élasticité } \\
\text { et de résistance } \\
\text { mécanique }\end{array}$ & Apparition des rides & $\begin{array}{l}\text { Désorganisation } \\
\text { du réseau matriciel } \\
\text { de collagène } \\
\text { et d'élastine }\end{array}$ & $\begin{array}{l}\text { Déséquilibre } \\
\text { de la balance } \\
\text { synthèse/dégradation } \\
\text { (augmentation } \\
\text { des MMP) }\end{array}$ & $\begin{array}{l}\text { Exfoliants chimiques } \\
(T C A, A H A, B H A)\end{array}$ \\
\hline Vasculaire & $\begin{array}{l}\text { Diminution } \\
\text { de la réactivité } \\
\text { vasculaire }\end{array}$ & $\begin{array}{l}\text { Défaut de thermorégulation } \\
\text { Fragilité à la pression }\end{array}$ & $\begin{array}{l}\text { Dysfonction } \\
\text { endothéliale }\end{array}$ & $\begin{array}{l}\text { Altération } \\
\text { du métabolisme du NO }\end{array}$ & $\begin{array}{l}\text { Composés sénolytiques } \\
\text { (dasatinib/quercétine) }\end{array}$ \\
\hline
\end{tabular}

Tableau I. Caractéristiques majeures du vieillissement chronologique de la peau et stratégies anti-âge.

de radicaux libres dans les tissus cutanés. En complément des filtres solaires, ils contribuent à limiter les effets les plus visibles des rayons UV, comme l'apparition de rides ou de taches pigmentées. Ils peuvent également stimuler la synthèse des collagènes et des fibres élastiques et limiter leur dégradation [22]. La vitamine A (ou rétinol) et ses dérivés ont également montré leur capacité à activer le métabolisme des collagènes tout en réduisant l'expression des collagénases, et à atténuer les effets du stress sur la peau en activant la voie du récepteur de I'EGF (epidermal growth factor) [30]. D'autres constituants majeurs de la matrice extracellulaire du derme, comme les protéoglycanes et les GAG, impactés par le vieillissement, peuvent être ciblés par des traitements anti-âge: c'est le cas, notamment, du C-xyloside qui stimule la production et le dépôt de ces composants, notamment de GAG [31], ainsi que des collagènes de la jonction dermo-épidermique [32]. Au niveau de l'épiderme, l'application d'exfoliants chimiques, comme l'acide trichloroacétique (TCA) ou des alpha/bêta-hydroxyacides (AHA ou BHA), permet de supprimer des couches cellulaires épidermiques, à des profondeurs variables selon les molécules utilisées, ce qui active la prolifération cellulaire et peut contribuer à restaurer l'architecture du tissu [33]. Les stratégies visant à améliorer la microcirculation cutanée chez les personnes âgées incluent l'activité physique régulière. Celle-ci peut contribuer à réduire le stress oxydant, ce qui se traduit par un accroissement de la vasodilatation dépendant de l'endothélium. Les approches médicamenteuses sont à base d'antioxydants, comme la vitamine $C$, qui augmente la vasodilatation liée à l'acétylcholine chez des sujets de plus de 60 ans [34], ou l'albumine qui améliore la réponse endothéliale et donc stimule la microcirculation cutanée [35].

La sénescence cellulaire est un autre mécanisme majeur qui participe à l'établissement de défauts fonctionnels liés à l'âge. L'accumulation délétère de cellules sénescentes est observée aussi bien dans le derme que dans l'épiderme de la peau de sujets âgés. Le traitement avec des composés ciblant les cellules sénescentes, ou sénolytiques, est donc une approche prometteuse qui s'est développée ces dernières années $[36,42](\rightarrow)$. Plusieurs sénolytiques ont été testés sur des modèles

$(\rightarrow)$ Voir la Chronique génomique de B. Jordan, $m / s n^{\circ} 10$, octobre 2018, page 885 et la Synthèse de $D$. Veret et J.M. Brondello, page 1135 de ce numéro

en culture ou des explants de peau : un extrait de la plante Solidago alpestris est capable de retarder l'entrée en sénescence de fibroblastes dermiques et améliore leur capacité à soutenir la reconstruction épidermique dans un modèle de culture en trois dimensions [37]; le composé $A B T 737^{2}$ cible les mélanocytes sénescents, qui exercent un effet paracrine délétère sur les kératinocytes de l'épiderme, ce qui permet de restaurer leur activité proliférative [38]; le sélénium appliqué sur des cultures de kératinocytes les protège de la sénescence réplicative et améliore les caractéristiques du tissu dans un modèle de peau reconstruite mimant le vieillissement [39]. Certains sénolytiques font maintenant l'objet d'essais cliniques. C'est le cas du duo dasatinib/quercétine dont l'efficacité a été testée dans une étude pilote de phase I : chez des individus diabétiques ayant reçu ce traitement par voie orale, une diminution du nombre de kératinocytes sénescents, exprimant les molécules p16 et p21, des inhibiteurs de kinases dépendant des cyclines, a été observée dans l'épiderme, associée à une augmentation du nombre

\footnotetext{
${ }^{2}$ Un inhibiteur de $\mathrm{Bcl}-\mathrm{xL}, \mathrm{Bcl}-2$ and $\mathrm{Bcl}-\mathrm{w}$, mimétique de $\mathrm{BH} 3$.
} 
de cellules de Langerhans. Au niveau sanguin, une réduction de cytokines pro-inflammatoires et des MMP typiques du SASP a également été détectée [40]. Les effets à long terme de ces traitements doivent maintenant être évalués sur un nombre plus important de patients.

\section{Conclusion}

La peau constitue un miroir de la physiologie générale : les anomalies structurales ou fonctionnelles détectées au niveau de la peau peuvent en effet alerter sur de possibles dysfonctionnements d'organes internes. Elle représente un organe de choix pour étudier les effets biologiques du vieillissement: organe visible dont les altérations sont perceptibles à l'œil nu, elle est facilement accessible pour des prélèvements ou des études cliniques. C'est un organe complexe, multifonctionnel et composé de nombreuses structures et types cellulaires en interaction les uns avec les autres. Une approche intégrée visant à une meilleure compréhension de ces interactions et de leurs perturbations avec l'âge est nécessaire pour espérer maintenir l'intégrité fonctionnelle de la peau au cours du vieillissement. $\diamond$

\section{SUMMARY}

Functional integrity of aging skin, from cutaneous biology to antiaging strategies

The skin is a sentinel organ making easily visible the passing of time. Chronological and environmental aging weakens skin structure and functions. The skin barrier, the elastic and mechanical properties of the cutaneous tissue as well as its vascular reactivity are impacted by aging. The barrier dysfunction in aged skin is caused by defects in epidermal keratinocytes renewal and differentiation notably linked to abnormal expression of microRNAs regulating cell death and autophagy. An abnormal balance between synthesis and degradation of matrix proteins modifies the mechanical properties of the dermis in aged skin. Finally, a reduction of the vascular reactivity linked to endothelial dysfunctions is observed in elderly people. These biological processes can be targeted by therapeutic approaches either topical or systemic, especially using anti-oxydants or senolytics. These antiaging strategies might contribute to restore, at least in part, the functional integrity of aged skin. $\diamond$

\section{LIENS D'INTÉRÊT}

Les auteurs déclarent n'avoir aucun lien d'intérêt concernant les données publiées dans cet article.

\section{RéFÉRENCES}

1. Lopez-Otin C, Blasco MA, Partridge L, et al. The hallmarks of aging. Cell $2013 ; 153: 1194-217$.

2. Giangreco $A$, Qin $M$, Pintar $J E$, Watt FM. Epidermal stem cells are retained in vivo throughout skin aging. Aging Cell $2008 ; 7: 250-9$.

3. Ge Y, Miao Y, Gur-Cohen $\mathrm{S}$, et al. The aging skin microenvironment dictates stem cell behavior. Proc Natl Acad Sci USA $2020 ; 117: 5339-50$.

4. Lehmann M, Canatelli-Mallat M, Chiavellini P, Goya RG. A hierarchical model for the control of epigenetic aging in mammals. Ageing Res Rev $2020 ; 62$ : 101134.

5. Muther C, Jobeili L, Garion M, et al. An expression screen for aged-dependent microRNAs identifies miR$30 \mathrm{a}$ as a key regulator of aging features in human epidermis. Aging (Albany NY) 2017 ; 9 : 2376-96.

6. Chevalier F, Rorteau J, Lamartine J. microRNAs in the functional defects of skin aging. In : Intech0pen (ed). Non-coding RNAs 2020.
7. Gu Y, Han J, Jiang C, Zhang Y. Biomarkers, oxidative stress and autophagy in skin aging. Ageing Res Rev 2020 ; 59 : 101036.

8. Vermeij WP, Alia A, Backendorf C. ROS quenching potential of the epidermal cornified cell envelope. J Invest Dermatol 2011 ; 131 : 1435-41.

9. Haydont V, Bernard BA, Fortunel NO. Age-related evolutions of the dermis: clinical signs, fibroblast and extracellular matrix dynamics. Mech Ageing Dev $2019 ; 177: 150-6$.

10. Shin JW, Kwon SH, Choi Jy, et al. Molecular mechanisms of dermal aging and antiaging approaches. Int J Mol Sci $2019 ; 20$.

11. Lago JC, Puzzi MB. The effect of aging in primary human dermal fibroblasts. PLoS One 2019; 14 : e0219165.

12. Gruber F, Kremslehner C, Eckhart L, Tschachler $\varepsilon$. Cell aging and cellular senescence in skin aging - Recent advances in fibroblast and keratinocyte biology. Exp Gerontol $2020 ; 130$ : 110780.

13. Lopes-Paciencia S, Saint-Germain $\varepsilon$, Rowell MC, et al. The senescenceassociated secretory phenotype and its regulation. Cytokine $2019 ; 117: 15-22$.

14. Terlecki-Zaniewicz L, Lammermann I, Latreille J, et al. Small extracellular vesicles and their miRNA cargo are anti-apoptotic members of the senescence-associated secretory phenotype. Aging (Albany NY) 2018; 10 : 1103-32.

15. Terlecki-Zaniewicz L, Pils V, Bobbili MR, et al. Extracellular vesicles in human skin: cross-talk from senescent fibroblasts to keratinocytes by miRNAs. J Invest Dermatol $2019 ; 139$ : 2425-36 e5.

16. Choi $\varepsilon$, Kil IS, Cho \&G. Extracellular vesicles derived from senescent fibroblasts attenuate the dermal effect on keratinocyte differentiation. Int J Mol Sci $2020 ; 21$.

17. Cracowski JL, Roustit M. Human skin microcirculation. compr physiol 2020 ; $10: 1105-54$

18. Bentov $\mathrm{i}$, reed $\mathrm{mj}$. the effect of aging on the cutaneous microvasculature. Microvasc Res $2015 ; 100: 25-31$.

19. Johnson JM, Minson CT, Kellogg DL Jr. Cutaneous vasodilator and vasoconstrictor mechanisms in temperature regulation. Compr Physiol $2014 ; 4$ : 33-89.

20. Matz RL, Andriantsitohaina R. Age-related endothelial dysfunction: potential implications for pharmacotherapy. Drugs Aging $2003 ; 20: 527-50$.

21. Cau SB, Carneiro FS, Tostes RC. Differential modulation of nitric oxide synthases in aging: therapeutic opportunities. Front Physiol $2012 ; 3: 218$.

22. Nusgens BV, Humbert $P$, Rougier $A$, et al. Topically applied vitamin $C$ enhances the mRNA level of collagens I and III, their processing enzymes and tissue inhibitor of matrix metalloproteinase 1 in the human dermis. Invest Dermatol $2001 ; 116: 853-9$.

23. Payne GW. Effect of inflammation on the aging microcirculation: impact on skeletal muscle blood flow control. Microcirculation 2006 ; 13 : 343-52.

24. El Assar M, Angulo J, Vallejo S, et al. Mechanisms involved in the aginginduced vascular dysfunction. Front Physiol $2012 ; 3: 132$.

25. Gaubert ML, Sigaudo-Roussel D, Tartas M, et al. Endothelium-derived hyperpolarizing factor as an in vivo back-up mechanism in the cutaneous microcirculation in old mice. J Physiol 2007 ; $585: 617-26$.

26. Fromy B, Lingueglia $\varepsilon$, Sigaudo-Roussel D, et al. Asic 3 is a neuronal mechanosensor for pressure-induced vasodilation that protects against pressure ulcers. Nat Med $2012 ; 18: 1205-7$.

27. Fouchard M, Misery L, Le Garrec R, et al. Alteration of pressure-induced vasodilation in aging and diabetes, a neuro-vascular damage. Front Physiol $2019 ; 10: 862$.

28. Fromy B, Sigaudo-Roussel D, Gaubert-Dahan ML, et al. Aging-associated sensory neuropathy alters pressure-induced vasodilation in humans. J Invest Dermatol $2010 ; 130: 849-55$

29. Gaubert-Dahan ML, Castro-Lionard K, Blanchon MA, Fromy B. Severe sensory neuropathy increases risk of heel pressure ulcer in older adults. J Am Geriatr Soc 2013 ; $61: 2050-2$.

30. Romana-Souza B, Silva-Xavier W, Monte-Alto-Costa A. Topical retinol attenuates stress-induced ageing signs in human skin ex vivo, throughEGFR activation viaEGF, but notERK andAP-1 activation. Exp Dermatol $2019 ; 28$ : 906-13.

31. Pineau N, Carrino DA, Caplan Al, Breton L. Biological evaluation of a new $\mathrm{C}$-xylopyranoside derivative ( $\mathrm{C}$-Xyloside) and its role in glycosaminoglycan biosynthesis. Eur J Dermatol $2011 ; 21: 359-70$.

32. Sok J, Pineau N, Dalko-Csiba M, et al. Improvement of the dermal epidermal junction in human reconstructed skin by a new c-xylopyranoside derivative. EurJ Dermatol 2008 ; $18: 297-302$.

33. Abdel-Motaleb AA, Abu-Dief $\varepsilon \varepsilon$, Hussein MR. Dermal morphological changes following salicylic acid peeling and microdermabrasion. J Cosmet Dermatol $2017 ; 16$ : e9-el4. 


\section{RÉFÉRENCES}

34. Taddei S, Virdis A, Ghiadoni L, et al. Age-related reduction of $\mathrm{NO}$ availability and oxidative stress in humans. Hypertension $2001 ; 38: 274-9$.

35. Aldecoa C, Llau JV, Nuvials X, Artigas A. Role of albumin in the preservation of endothelia glycocalyx integrity and the microcirculation: a review. Ann Intensive Care $2020 ; 10: 85$.

36. Jordan B. La sénescence en passe d'être vaincue ? Med Sci (Paris) 2018 ; 34 : 885-90.

37. Lammermann I, Terlecki-Zaniewicz L, Weinmullner R, et al. Blocking negative effects of senescence in human skin fibroblasts with a plant extract. NPJ Aging Mech Dis $2018 ; 4: 4$

38. Victorelli S, Lagnado A, Halim J, et al. Senescent human melanocytes drive skin ageing via paracrine telomere dysfunction. EMBOJ 2019 ; 38 : e101982.

39. Jobeili L, Rousselle P, Beal D, et al. Selenium preserves keratinocyte stemness and delays senescence by maintaining epidermal adhesion. Aging (Albany NY) 2017; 9 : 2302-15.
40. Hickson LJ, Langhi Prata LGP, Bobart SA, et al. Senolytics decrease senescent cells in humans: Preliminary report from a clinical trial of Dasatinib plus Quercetin in individuals with diabetic kidney disease. EBioMedicine 2019; $47: 446-56$.

41. Boismal F, Serror K, Dobos G, et al. Vieillissement cutané : physiopathologie et thérapies innovantes. Med Sci (Paris) $2020 ; 36: 1163-72$.

42. Veret $D$, Brondello JM. Sénothérapies : avancées et nouvelles perspectives cliniques. Med Sci (Paris) 2020 ; $36: 1135-42$.

\section{TIRÉS À PART}

J. Lamartine

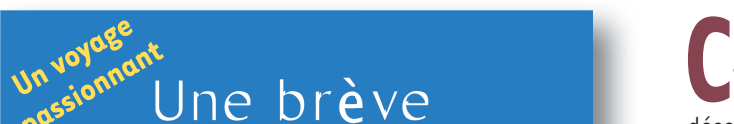
histoire du vaisseau sanguin et lymphatique

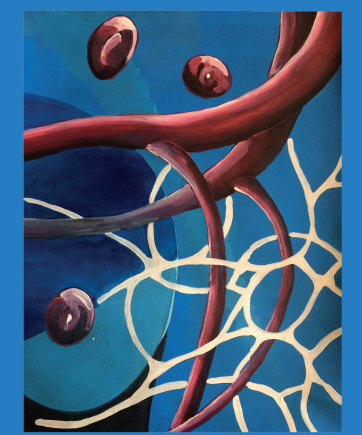

ANDREAS BIKFALVI

livre, intéressant et lisible à la fois pour le spécialiste et le grand public, apporte des observations originales et nouvelles concernant l'angiogenèse, et notamment l'histoire des différentes découvertes, et discute les aspects et les concepts plus généraux en les plaçant dans le contexte de la philosophie des sciences.

Facile à lire, bien illustré, cet ouvrage cherche à comprendre et à faire comprendre les enjeux de la recherche sur l'arbre vasculaire en développement et en pathologie. II intéressera non seulement les étudiants et post-doctorants en biologie, mais aussi les chercheurs actifs dans ce domaine de recherche ainsi que toute personne intéressée par la biologie et la médecine et par l'histoire des sciences. Un voyage passionnant à travers l'histoire et les concepts les plus actuels concernant les recherches sur le vaisseau sanguin.

Andreas Bikfalvi est Professeur à l'université de Bordeaux et Directeur d'une unité de recherche Inserm sur le cancer et la biologie vasculaire. Il est, par ailleurs, membre senior de l'Institut Universitaire de France (IUF) et reconnu internationalement pour ses recherches dans le domaine de l'angiogenèse tumorale.

À retourner à EDP Sciences, 17, avenue du Hoggar, 91944 Les Ulis Cedex, France Tél. : 0149856069 - Fax : 0149850345 - $\varepsilon$-mail : françois.flori@edpsciences.org

NOM :

Prénom :

Adresse:

Code postal :

Ville :

Pays :

Fonction :

Je souhaite recevoir

Une brève histoire de vaisseau : $25 €+3 €$ de port $=28 € \Pi C$

en . exemplaire, soit un total de $€$

$\square$ Par chèque, à l'ordre de EDP Sciences

$\square$ Par carte bancaire :

$\square$ Visa $\square$ Eurocard/Mastercard

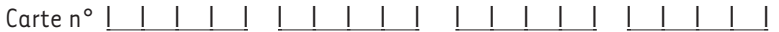

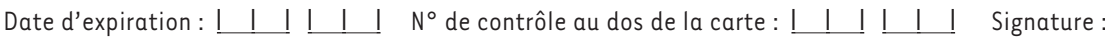

